\title{
Speckle Statistics of Localized Waves
}

\author{
A.A. Chabanov (University of Texas, San Antonio)
}

\author{
A. Peña \\ A. Genack
}

\$ NSF 


\title{
Speckle Statistics of Localized Waves
}

\section{A.A. Chabanov (University of Texas, San Antonio)}

\author{
A. Peña \\ A. Genack
}

\$ NSF 


\section{Wave propagation in disordered media}

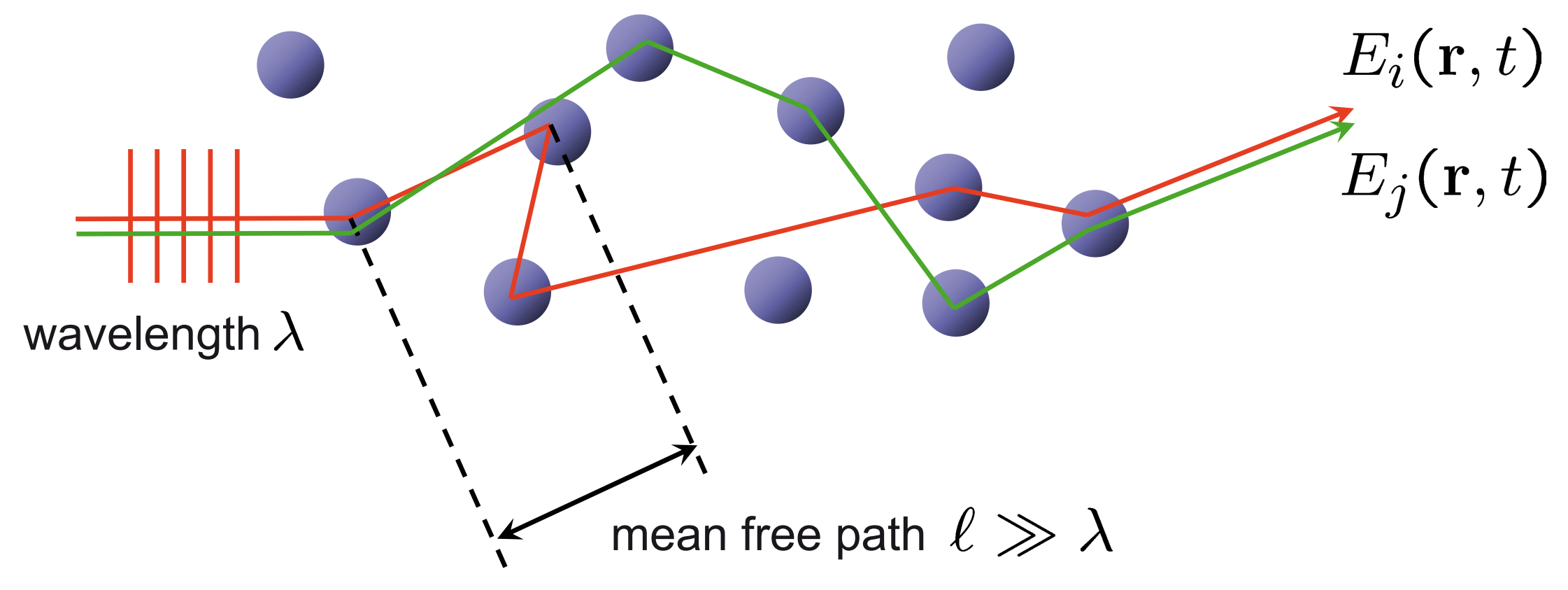

Field $E(\mathbf{r}, t)=\sum_{i} E_{i}(\mathbf{r}, t)$

Intensity $I(\mathbf{r}, t)=|E(\mathbf{r}, t)|^{2}=\sum_{i, j} E_{i}(\mathbf{r}, t) E_{j}^{*}(\mathbf{r}, t)$ 


\section{Wave propagation in disordered media}

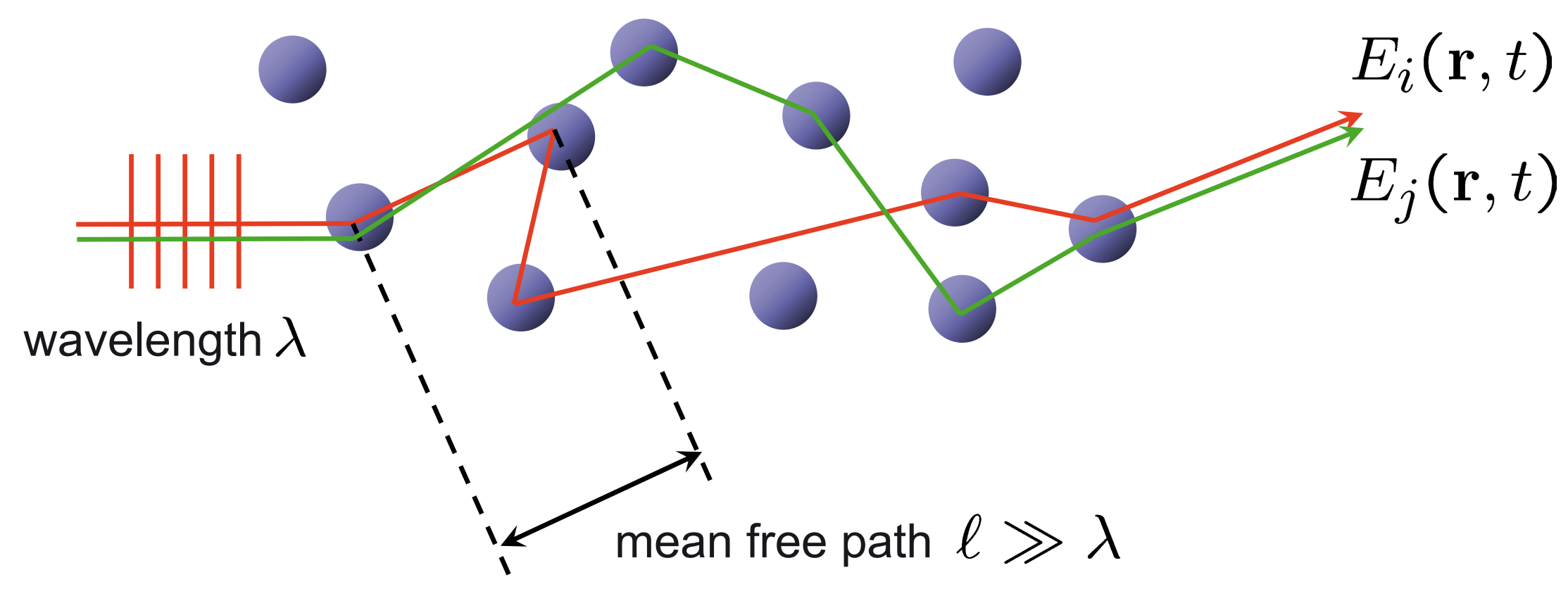

Average intensity:

$$
\langle I(\mathbf{r}, t)\rangle=\sum_{\substack{i \\ \text { independent paths }}}\left\langle\left|E_{i}(\mathbf{r}, t)\right|^{2}\right\rangle+\sum_{i \neq j} \overline{\left\langle E_{i}(\mathbf{r}, t)\right.}
$$




\section{Weak localization and non-Gaussian statistics}
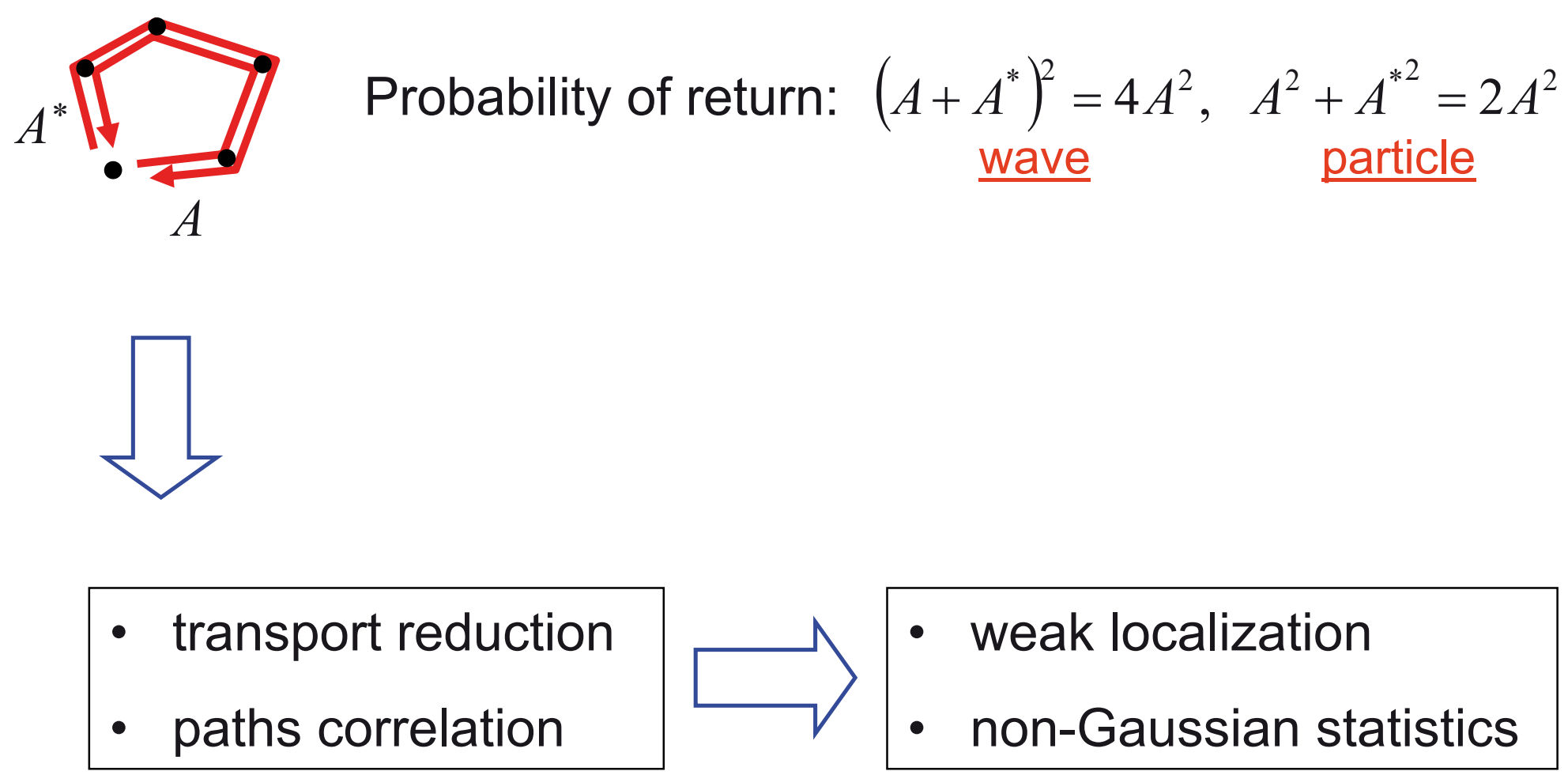


\section{Transmission coefficients}

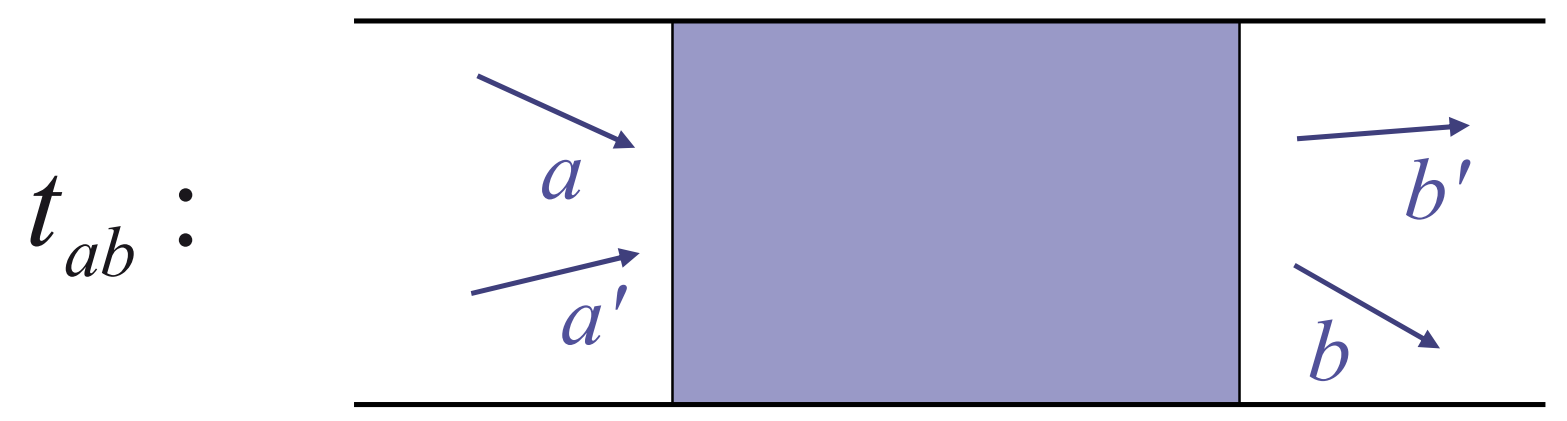

$T_{a b}=\left|t_{a b}\right|^{2} \quad$ Transmitted intensity = speckle intensity

$T_{a}=\sum_{b} T_{a b} \quad$ Total transmission $=>$ brightness

$T=\sum_{a} T_{a}=\sum_{a b} T_{a b} \quad$ Transmittance $=$ conductance 


\section{Random matrix theory}

$$
\begin{gathered}
t=U\left(\begin{array}{ccc}
\sqrt{\tau_{1}} & & 0 \\
& \cdots & \\
0 & & \sqrt{\tau_{N}}
\end{array}\right) V, \quad t t^{\dagger}=\left(\begin{array}{ccc}
\tau_{1} & & 0 \\
& \cdots & \\
0 & & \tau_{N}
\end{array}\right) \\
P\left\{\tau_{n}\right\}=>P\left(T_{a b}\right), P\left(T_{a}\right), P(T)
\end{gathered}
$$




\section{Non-Gaussian statistics of transmission}

$$
\begin{gathered}
t_{a b}=\Sigma_{n} u_{a n} \sqrt{\tau_{n}} v_{n b}=\sqrt{\Sigma_{n}\left|u_{a n}\right|^{2} \tau_{n}} \times t_{a b}^{\prime}=\sqrt{T_{a}} \times t_{a b}^{\prime} \\
\text { AAC \& Genack, PRA (2005) } \\
T_{a b}=T_{a} \times\left|t_{a b}^{\prime}\right|^{2} \\
P_{a}\left(T_{a b}\right)=\frac{1}{T_{a}} \exp \left(-T_{a b} / T_{a}\right)=>P\left(T_{a b}\right)=\int_{0}^{\infty} \frac{d T_{a}}{T_{a}} P\left(T_{a}\right) \exp \left(-T_{a b} / T_{a}\right) \\
\left\langle T_{a b} T_{a^{\prime} b^{\prime}}\right\rangle=\left\langle T_{a} T_{a^{\prime}}\right\rangle \times\left\langle\left|t_{a b}^{\prime}\right|^{2}\left|t_{a^{\prime} b^{\prime}}^{\prime}\right|^{2}\right\rangle
\end{gathered}
$$

Kogan \& Kaveh, PRB (1995) 


\section{Probability distribution of total transmission}

$$
\begin{gathered}
P\left(T_{a}\right)=L^{-1}\{F(z)\}, \\
F(z)=\exp \left[-\langle g\rangle \ln ^{2}(\sqrt{1+z /\langle g\rangle}+\sqrt{z /\langle g\rangle})\right], \quad g=\Sigma_{n} \tau_{n}
\end{gathered}
$$

Nieuwenhuizen \& Van Rossum, PRL (1995)

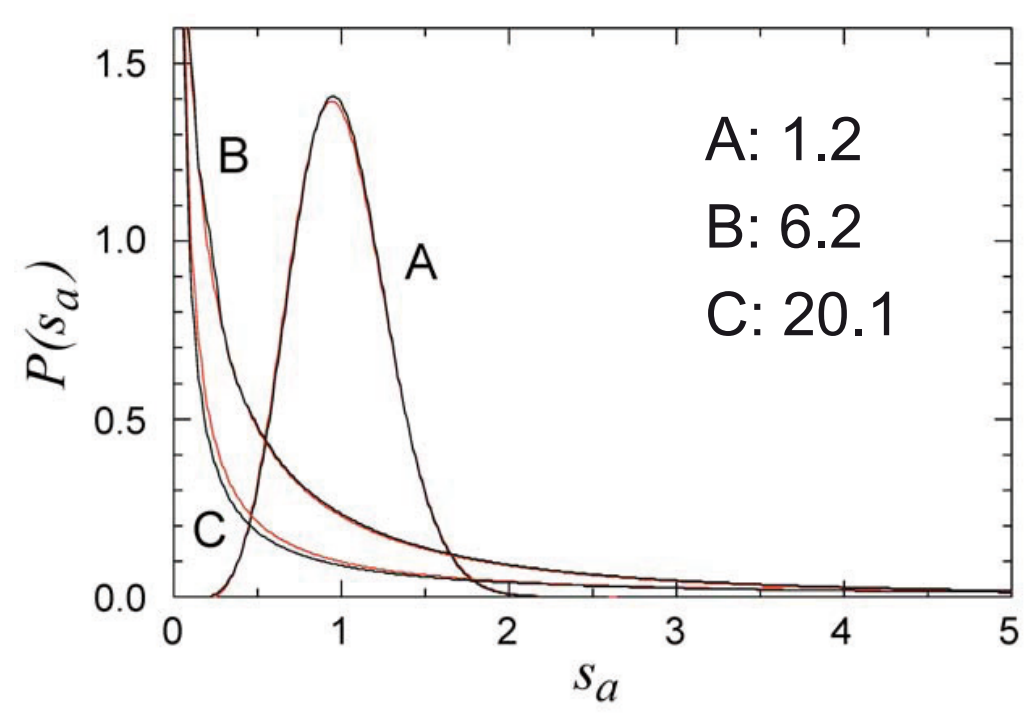

$$
\operatorname{var}\left(T_{a}\right)=2 / 3\langle g\rangle
$$

AAC \& Genack, PRA (2005) 


\section{Intensity correlation with polarization}

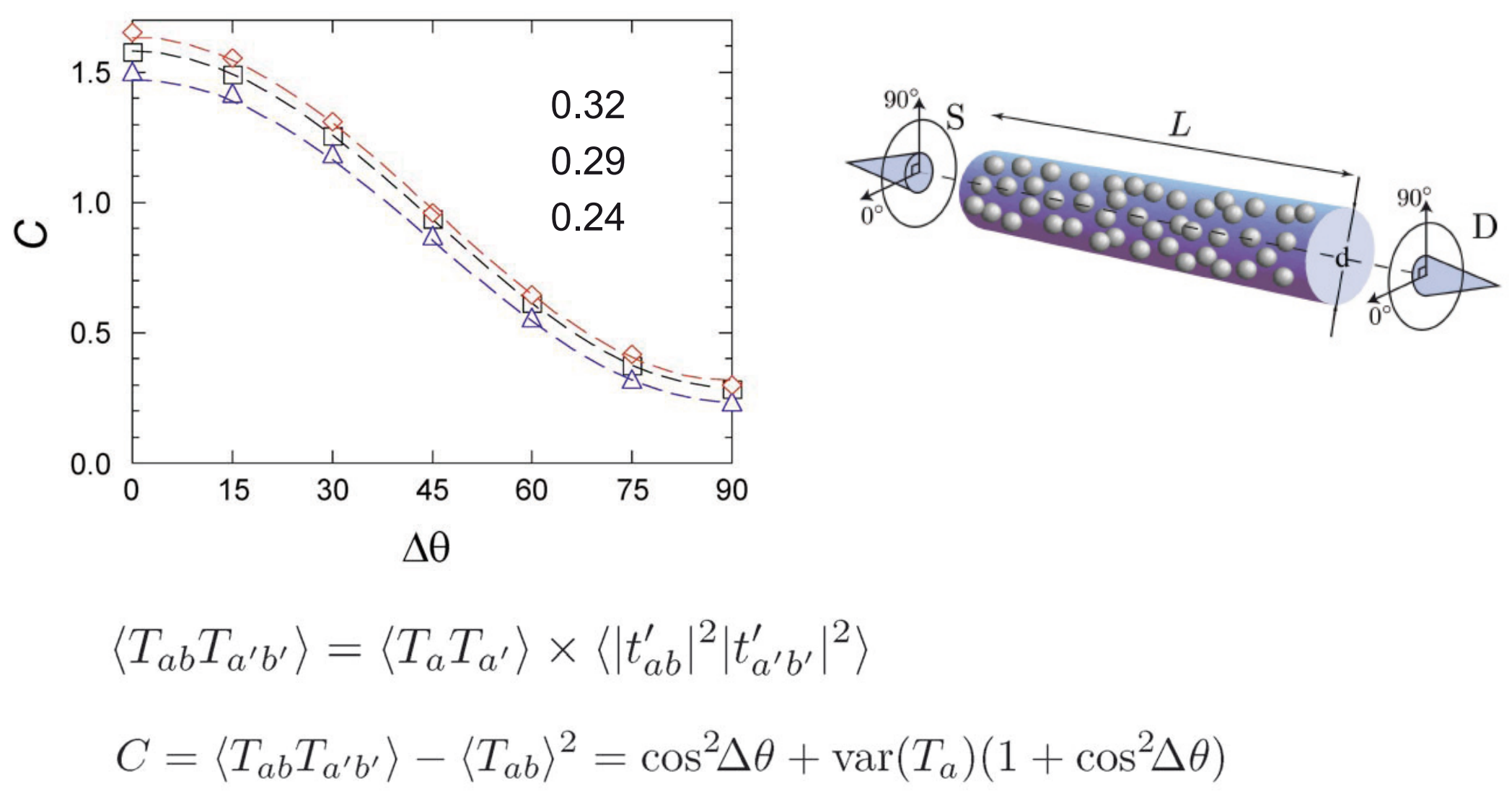

AAC, Hu \& Genack, PRL (2004) 


\section{Speckle statistics of localized waves}

Q: How speckle pattern of localized waves looks like?

Q: How different it is from that of extended waves?

Q: What we can learn from speckle statistics of localized waves? 


\section{Experimental setup}

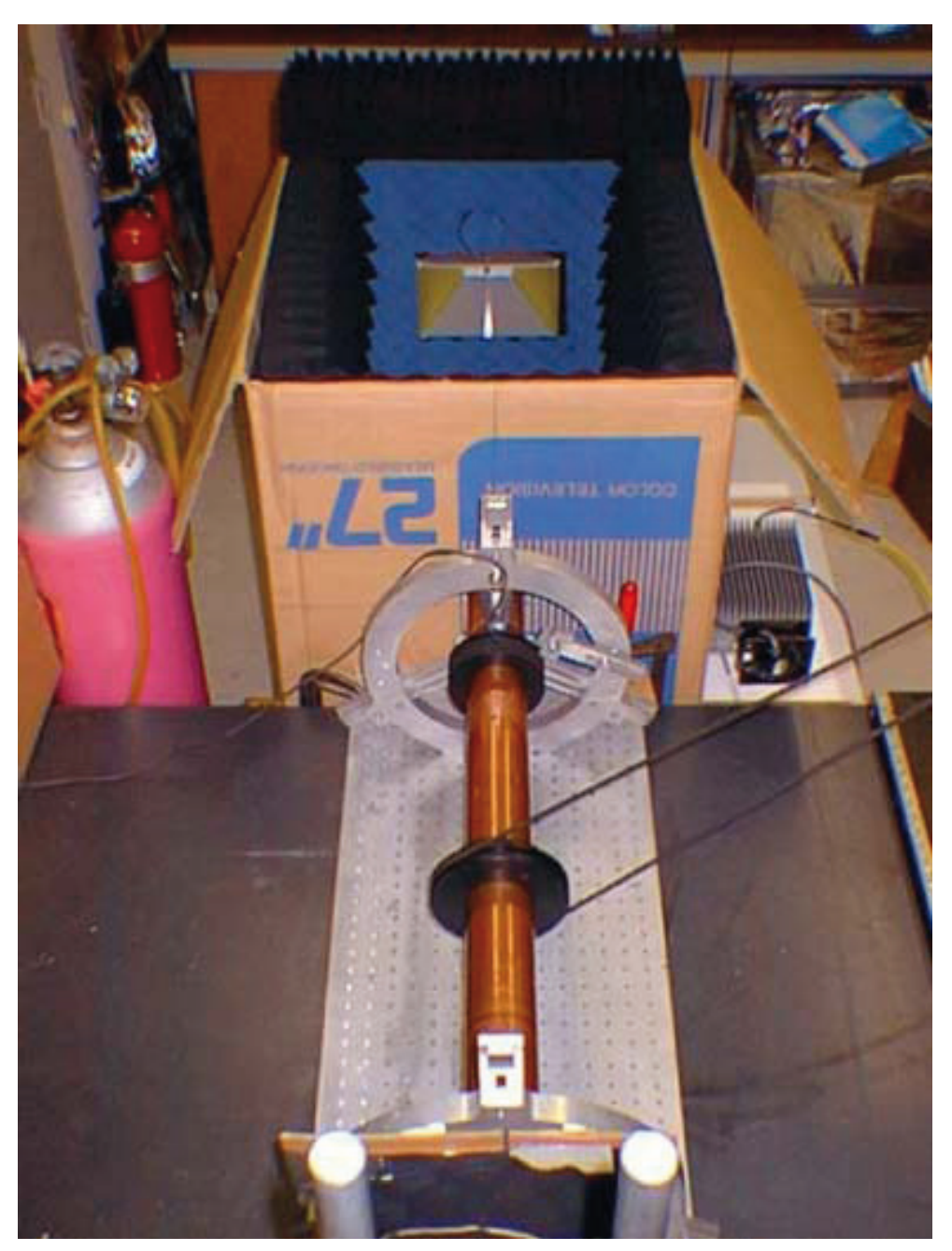




\section{Alumina sample}

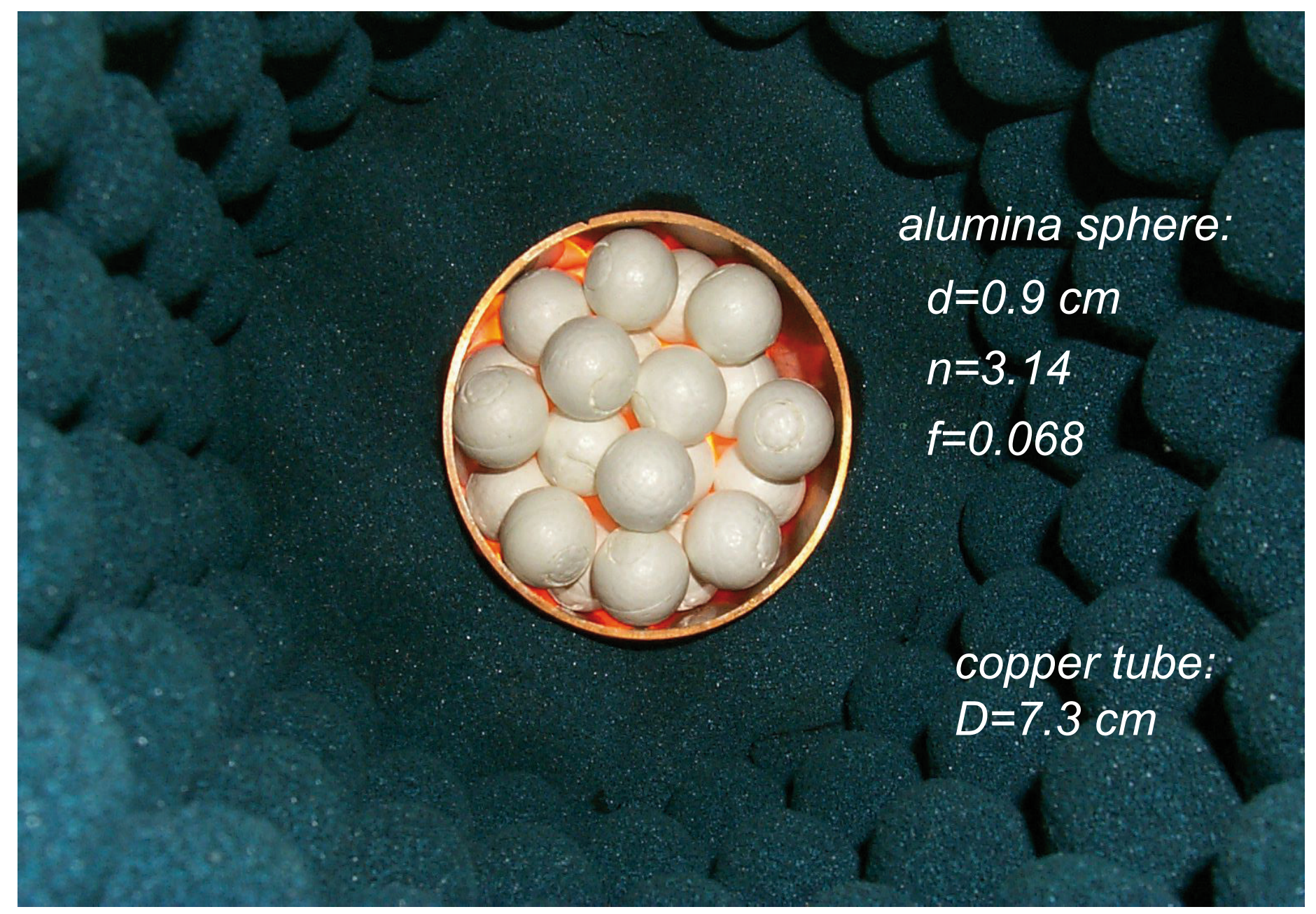




\section{Correlations with polarization}

$$
\begin{aligned}
C\left(\Delta \theta_{S}, \Delta \theta_{D}\right)= & \cos \left(\Delta \theta_{S}\right) \cos \left(\Delta \theta_{D}\right)+A_{2}\left(\cos \left(\Delta \theta_{S}\right)+\cos \left(\Delta \theta_{D}\right)\right)+ \\
& +A_{3}\left(1+\cos \left(\Delta \theta_{S}\right)+\cos \left(\Delta \theta_{D}\right)+\cos \left(\Delta \theta_{S}\right) \cos \left(\Delta \theta_{D}\right)\right)
\end{aligned}
$$

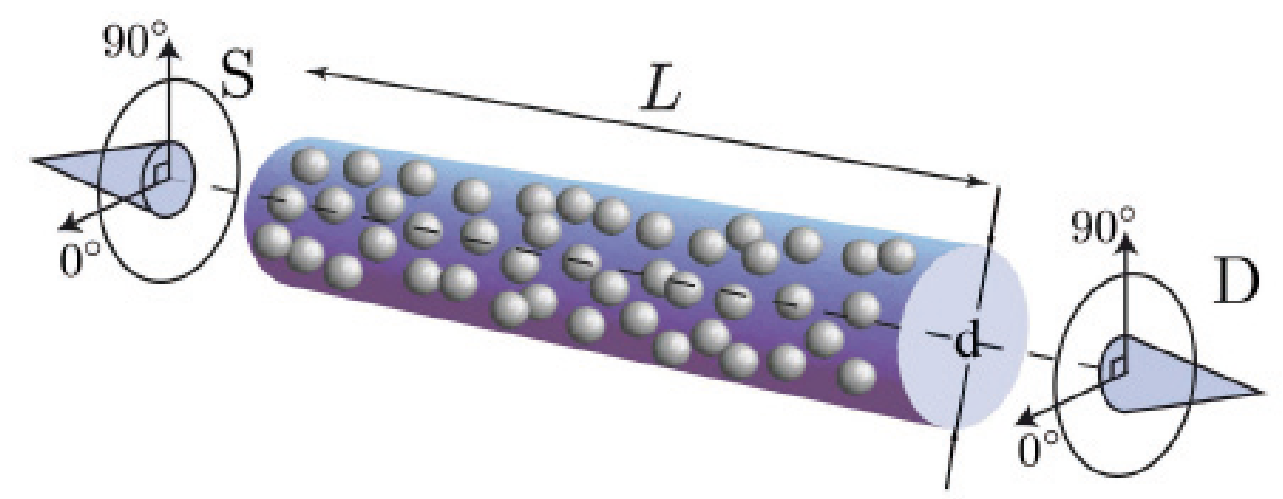

$$
C(90,90)=A_{3}, \quad C(0,90)=C(90,0)=A_{2}+2 A_{3}
$$

Chabanov et al, PRL 92, 173901 (2004) 


\section{Intensity correlation of localized waves}

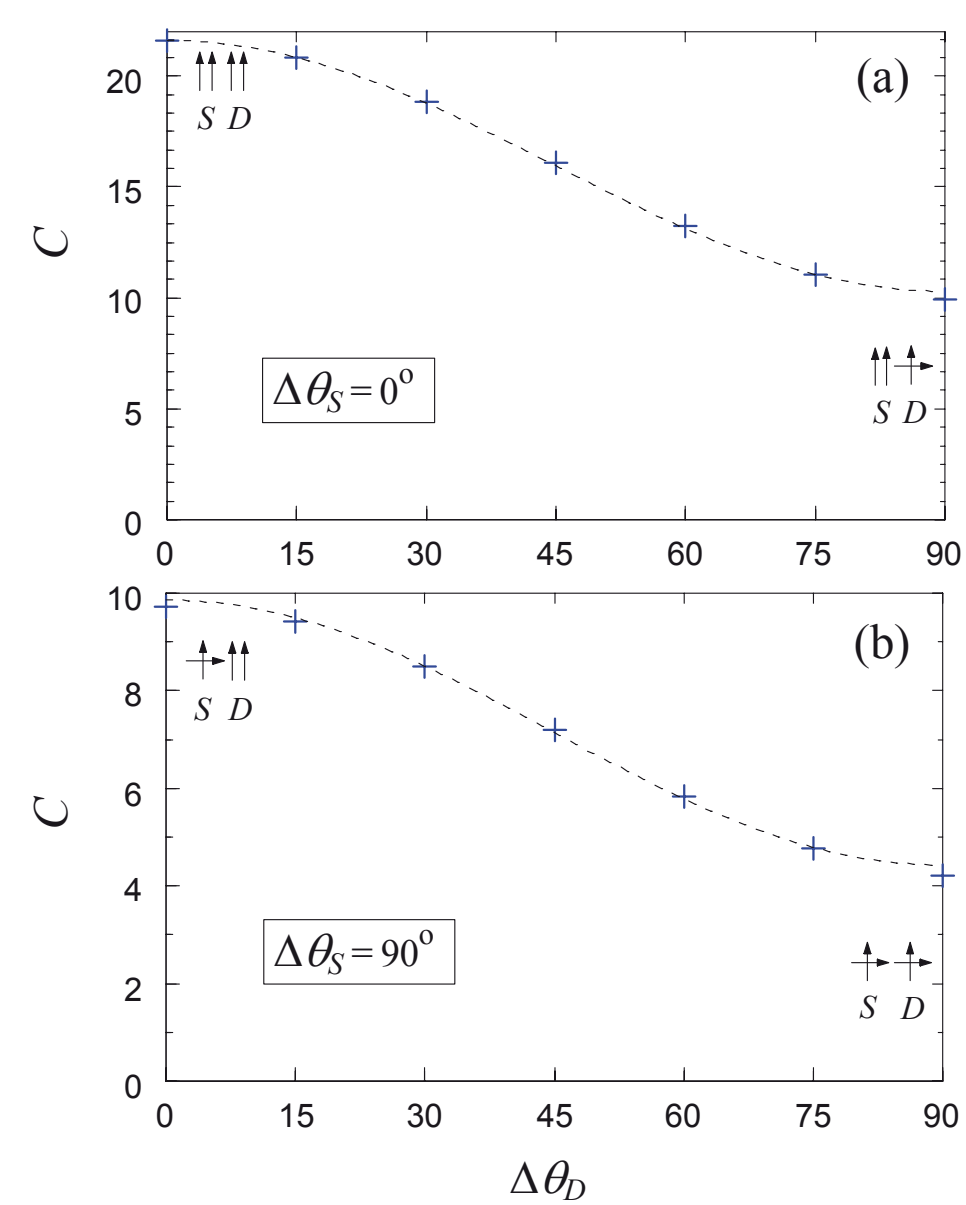

Alumina localized sample, $L=4 \xi$

From fit: $A_{2}=1.00 \pm 0.02$

$$
A_{3}=4.38 \pm 0.04
$$




\section{Intensity correlation of localized waves}

$$
\begin{aligned}
& \frac{\left\langle T_{a b} T_{a^{\prime} b^{\prime}}\right\rangle}{\left\langle T_{a b}\right\rangle^{2}}=C\left(\Delta \theta_{S}, \Delta \theta_{D}\right)+1=\left(1+A_{3}\right)\left(1+\cos \left(\Delta \theta_{S}\right)\right)\left(1+\cos \left(\Delta \theta_{D}\right)\right) \\
& \left\langle I^{2}\right\rangle=4\left(1+A_{3}\right) \quad \Delta \theta_{\mathrm{D}}=0, \Delta \theta_{\mathrm{S}}=0 \\
& \left\langle I_{1} I_{2}\right\rangle=1+A_{3} \quad \Delta \theta_{\mathrm{D}}=90, \Delta \theta_{\mathrm{S}}=90 \\
& \frac{\left\langle I^{2}\right\rangle}{\left\langle I_{1} I_{2}\right\rangle}=4
\end{aligned}
$$




\section{Intensity correlation of localized waves}

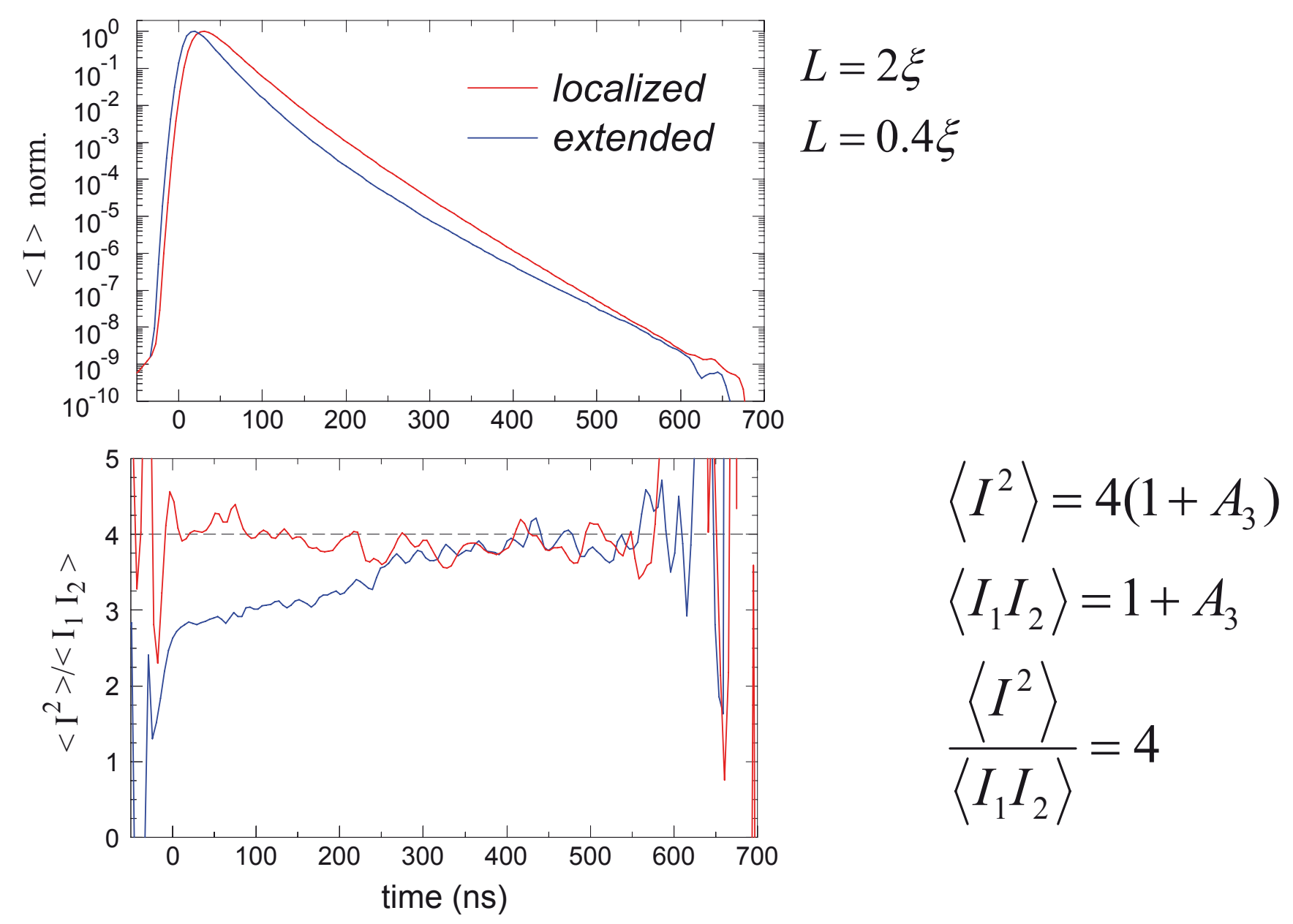




\section{Statistics of transmission quantities in localized regime}

$$
\begin{gathered}
T_{a b}=\left|u_{a 1}\right|^{2} \tau_{1}\left|v_{1 b}\right|^{2}, \\
T_{a}=\left|u_{a 1}\right|^{2} \tau_{1}, \\
T=\tau_{1} .
\end{gathered}
$$

We now switch to the normalized quantities, $s_{a b}=T_{a b} /\left\langle T_{a b}\right\rangle, s_{a}=T_{a} /\left\langle T_{a}\right\rangle$, and $s=T /\langle T\rangle$.

$$
\begin{gathered}
\left\langle s_{a b}^{n}\right\rangle=(n !)^{2}\left\langle s^{n}\right\rangle, \\
\left\langle s_{a}^{n}\right\rangle=n !\left\langle s^{n}\right\rangle .
\end{gathered}
$$

These lead to the relations between the statistics of the transmission quantities,

$$
\begin{gathered}
P\left(s_{a}\right)=\int_{0}^{\infty} \frac{d s}{s} P(s) \exp \left(-s_{a} / s\right), \\
P\left(s_{a b}\right)=\int_{0}^{\infty} \frac{d s_{a}}{s_{a}} P\left(s_{a}\right) \exp \left(-s_{a b} / s_{a}\right)=2 \int_{0}^{\infty} \frac{d s}{s} P(s) K_{0}\left(2 \sqrt{s_{a b} / s}\right),
\end{gathered}
$$

Correlations: $\quad \Gamma_{s_{a b}}\left(\Delta \theta_{\text {in }}, \Delta \theta_{\text {out }}\right)=\left\langle s^{2}\right\rangle\left(1+\cos ^{2}\left(\Delta \theta_{\text {in }}\right)\right)\left(1+\cos ^{2}\left(\Delta \theta_{\text {out }}\right)\right)$ 


\section{Statistics of transmission quantities in localized regime}

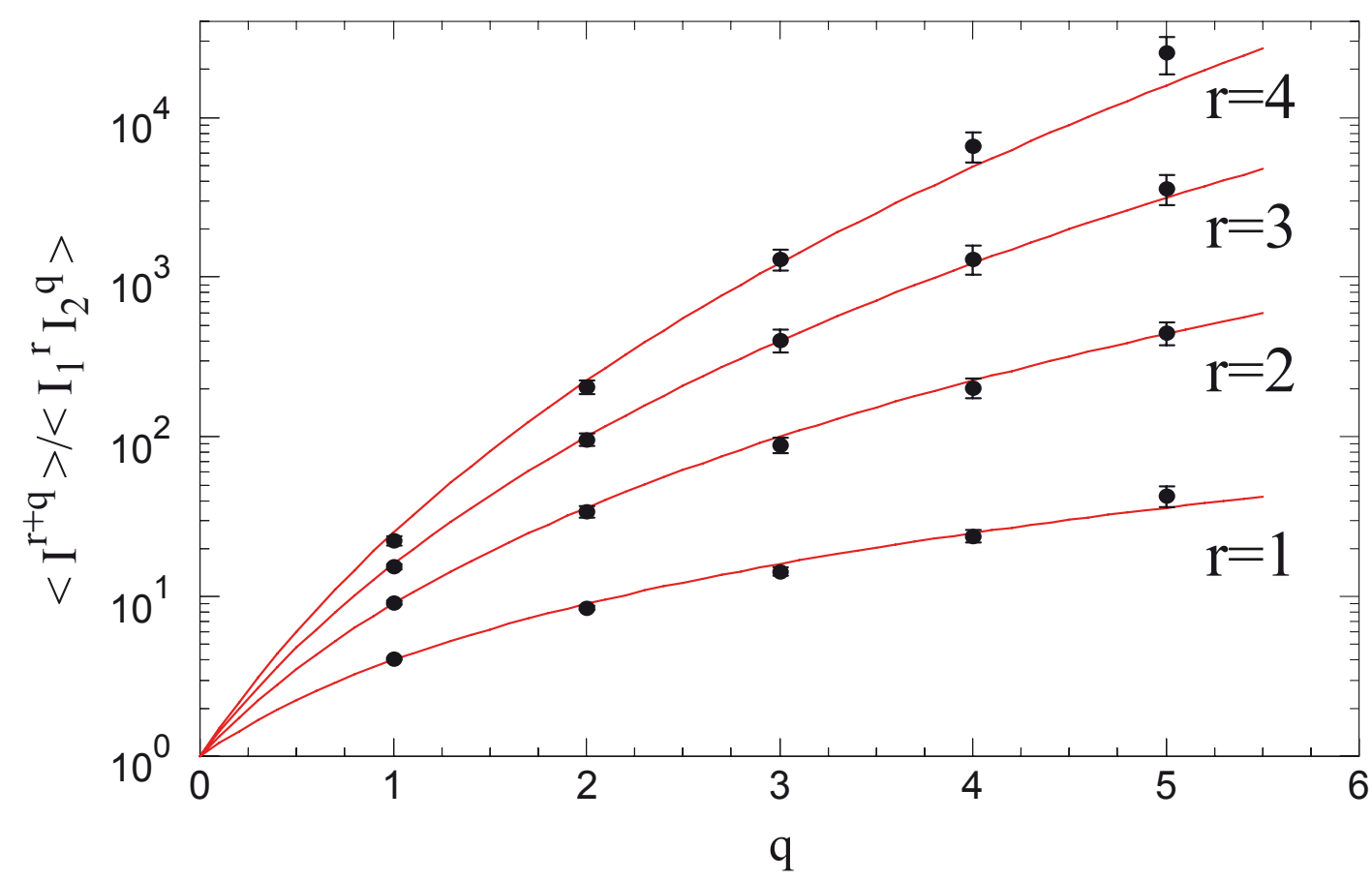

$$
\frac{\left\langle I^{r+q}\right\rangle}{\left\langle I_{1}^{r} I_{2}^{q}\right\rangle}=\left(\frac{(r+q) !}{r ! q !}\right)^{2}
$$




\section{Statistics of conductance in localized regime}

$$
\begin{gathered}
\ln \left(s_{a b}\right)=\ln \left|u_{a 1}\right|^{2}+\ln (s)+\ln \left|v_{1 b}\right|^{2} \\
F_{\ln \left(s_{a b}\right)}(\omega)=F_{\ln (s)}(\omega) \Gamma(1+i \omega)^{2} \\
P[\ln (s)]=\int_{-\infty}^{+\infty} d z \exp [i z \ln (s)] F_{\ln (s)}(z)
\end{gathered}
$$




\section{Statistics of conductance in localized regime}

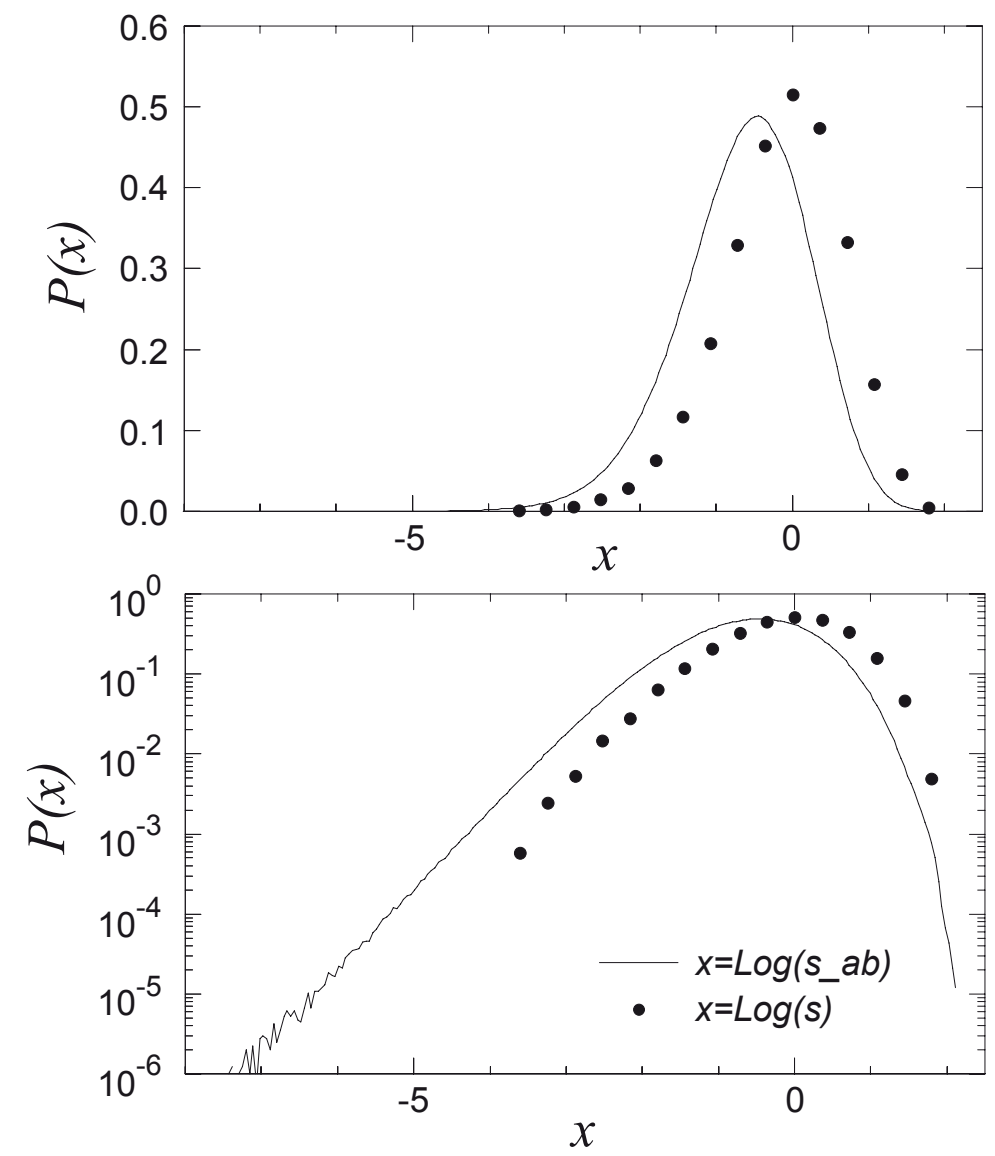




\section{Summary}

- In a given quasi-1D random configuration, the statistics of transmitted field is Gaussian for both diffusive and localized waves; non-Gaussian mesoscopic field statistics arise in ensemble of configurations due to mesoscopic fluctuations of transmission.

- For localized waves, transmission statistics is rather simple: only statistics of conductance matters, whereas statistics of total transmission and speckles follow. The distribution of conductance can be easily determined from transmission or speckle intensity distributions

- The factorization of the speckle statistics can be used as indication of the localization threshold, because only one transmission contributes. 